\title{
Development decision centers - a strategy to improve development decision-making
}

\author{
D. Splettstoesser \\ Dept. of General Management, University of Dar Es Salaam \\ P.O. Box 35046, Dar Es Salaam, Tanzania \\ E-mail:splett@unidar.gn.apc.org
}

\begin{abstract}
Political, economic and social development requires 'good governance' and intelligent decisionmaking. Development decision centers (DDC) equipped with adequate information technology (IT) can help to improve development decisions, increase effectiveness and efficiency of decision conferences and support democratization and political stability.

DDC provide IT-based facilities for brainstorming, discussion, organization and evaluation of ideas, objectives, strategies, projects, etc. They can be integrated into community information centers which are recognized as a 'multisectoral concept' for improving development. They can also be established within government agencies, business corporations, universities and other institutions of higher learning to offer assistance in solving complex development problems that require close collaboration of management and expert teams.

The paper analyzes political, socio-cultural and economic development problems, and explains typical steps of development decision-making, using a group support system in the DDC, the author has established at the University of Dar es Salaam, Tanzania. It is believed to be the first such center in Africa.

In view of the increasing globalization of trade and international cooperation, DDC can play an important role in the concept of the International Office of the Future. They help to integrate developing countries into the world economy and enhance intercultural understanding while, at the same time, improving transparency, accountability and productivity of development planning.
\end{abstract}

\section{Keywords}

Development planning, management of development projects, development cooperation, group techniques, group decision-making, group support systems, group productivity, computer-supported collaborative work, electronic meetings 


\section{PROBLEMS OF DEVELOPMENT DECISION-MAKING}

Development decision-making is concerned with political, economic and social progress in developing countries. Significant differences in problems and decision processes, depending on the political framework and socio-cultural background, exist in those countries. However, in general, there seem to be increasing difficulties in achieving development progress. Factors, such as lack of development-orientation and political stability, insufficient strategic planning, high level of official corruption, mass poverty, inadequate infrastructure, etc. affect almost all developing countries, particularly in Africa.

In view of these difficulties and the overall complexity of development, one may sometimes forget that most development problems are man-made and influenced by decisions that can be improved considerably. Information technology (IT) is expected to play a key role in implementing and sustaining such improvements in development decision-making, if appropriate strategies for exploiting its potential are found.

The subsequently discussed idea of development decision centers (DDC) appears to be a promising strategy. It is based on the author's experience as a lecturer, consultant and manager in East Africa since 1981. DDC are expected to substantially improve development decision-making, as they support the concept of 'good governance' through ensuring accountability, transparency, participation, productivity and decision quality. DDC may become a core facility within community information centers, providing an 'electronic meeting room' with notebook computers for each participant, a workstation for the meeting facilitator/chauffeur, a printer and a video projector to output the meeting results, network and communication software, and a group support system to assist in activities, such as brainstorming, discussing, organizing, evaluating and reporting of development programs, projects, funding, etc.

Development decisions are taken by governments, international financing institutions, nongovernmental organizations, public and private corporations, foundations, communities, etc. in developing or donor countries. DDC can help in any such decision situation. In this paper, they are suggested as a strategy for developing countries, as they do not only assist in development decision-making itself, but also contribute to enhance a country's information infrastructure and affect the political, cultural and economic environs of development decision-making.

\subsection{Political and Cultural Aspects}

\section{The Development Dilemma: 'Nation-Building' and National Disintegration}

Development policy and decision-making started more than 30 years ago. It aimed at improving the economic and social situation of people in developing countries. In Africa, those countries typically had been colonies of European nations until the late 1950s or early 1960s. They had not only to overcome major problems from their colonial past, but also had to integrate numerous ethnic communities of different languages and cultures. To help these groups agree to a common development agenda, the newly independent governments considered 'nation-building' an important prerequisite for development.

At the same time, i.e. from about 1960 onwards, a number of West-European nations, including the former colonial powers that played a key role in shaping development policy, had begun to reorient themselves from a narrow national view of development to a wider European perspective. This led to the establishment of the European Community. From the beginning of the 1980s, however, Europeans began to realize that neither the European Community nor the national 
governments were able to adequately fulfill the citizens' expectations with regard to growth, stability and employment. European administrative structures and procedures were increasingly criticized and considered an ineffective, unmanageable and costly bureaucracy. A gradual and rather unexpected renaissance of regions began. Other factors contributing to this shift were the rediscovery of original cultural values and identity, and, not least, 'glasnost' and the 'information revolution'. Among the consequences were not only decreasing enthusiam for European unity, but also the dramatic demise of communism, the Balkan tragedy, and increasing suspicion against all forms of central (national) administration.

Inevitably, these changes of political structures and cultural orientation also had an impact on development and developing countries. In North Africa, increasing tendencies for fundamentalism as evident in Algeria, Libya, and Egypt led to adverse development conditions. In Sub-Saharan Africa, civil war and irreconcilable conflicts between ethnic groups, living within national boundaries that were determined more than 100 years ago without regard to cultural differences, made reasonable development almost impossible. Examples for this can be found all over East and Central Africa, particularly in Somalia, Rwanda and Burundi. Hutus and Tutsis in Rwanda and Burundi will hardly ever be able to devise a common development strategy in a national context. Equally, national development efforts are unimaginable in Sudan, where the central government since more than 10 years fights a rebel movement that aims at establishing an independent state in the South. Even in Kenya, that appears in a relatively fortunate position when compared to its neighbors, national cooperation among the different ethnic groups is on the decline and 'majimboism' (regionalism) is growing.

Under these circumstances, sustainable economic and human development requires more efforts than national governments have been able to make. It requires particularly 'more local participation in the design and implementation of development programs', as the former president of the World Bank noted (WorldBank, 1992). This implies that development should ideally not be pursued as a national, government-initiated task, but as a community-initiated and locally managed effort, taking into consideration the accumulated knowledge and understanding of the people concerned.

\section{Lack of 'Good governance'}

Development requires 'good governance' which in essence means democratic decision-making, respect for human rights and the rule of law, efficiency, accountability, transparency in government and public administration. This is rarely achieved. While there is no one best way to 'good governance' and sustainable development (Blunt, 1995), there are universal best practices, like participative approaches to policymaking and decentralization, that deserve to be pursued.

Governments in many African countries have yet to reform decision-making processes that were devised under post-colonial, rather authoritarian regimes. There is frequently a considerable lack of political will and perhaps of understanding on the side of the ruling elites to do that. In recent years, several governments in Africa had to be pressed hard to accept more democracy and participation in policymaking. Frequently, there has been an attempt to preserve existing power structures and resist donor demands for changes. However, this resistance may have also been caused by different ideas about individual and collective freedom. In collectivist societies (Hofstede, 1983), where 'ingroups', for example tribes, play an important role, individual rights are usually less important than in individualist societies. This explains a rather hesitant position towards political demands for more individual freedom. The resulting lack of democratic structures and procedures, particularly the insufficient involvement of large parts of the population in development planning and program implementation, has most likely contributed to the fact that, despite all development aid, the economic and social gap between developed countries in Europe and North America and African 
developing countries has widened dramatically over the last twenty years.

Another factor hampering development, particularly in Africa, has been the rampant corruption on all levels of public life. While corrupt practices have become acceptable in many societies and have typically been acknowledged in taxation laws to the degree of deductibility of bribes from income tax, they could still be contained by strong mechanisms to ensure accountability and transparency of government and public institutions. Where such mechanisms are not yet in place or poorly enforced, like in many African countries, corruption spread like a contagious disease. Its devastating effects on development, resulting frequently from irrational and shortsighted development decision-making (Sabuni, 1996) are clearly visible. Despite the fact that codes of ethic for members of governments and civil service were introduced, and new laws to severely punish corruption were enacted in several countries, the practice went on unabated, since the underlying economic and societal conditions remained unchanged and sufficient controls, including a free press, were not firmly established.

As a legacy from colonialism, centralization of information and policymaking power is still high in most African developing countries. Decentralization efforts have started in some countries, but no acceptable level of participative policymaking and accessibility to public services, facilities and information has yet been reached. Most rural areas have been neglected in development efforts. This has led to huge information and communication deficits in these areas, further discrepancies between urban and rural living conditions and the resulting increase of migration to urban centers. Strategies to reverse this trend by strengthening rural development opportunities and allow rural dwellers to easily communicate not only with their own government institutions, legislative and judicial bodies, but also with other national and international partners will most likely facilitate 'good governance' and accelerate development.

\section{Participative culture and 'Power distance'}

Development decision-making requires involvement and participation of the main stakeholders. One could assume that this can be easily achieved in collectivist societies which have been typical for African developing countries. Such societies are characterized by tight integration of people who are supposed to look after the interests of the 'ingroup' and have no other opinions and beliefs than this group (Hofstede, 1983). Tribalism which is ubiquitous in Africa gives ideal examples for such a society. The resulting high level of integration is, however, inevitably restricted to the members of the 'ingroup'.

For development decision-making, members of all concerned communities would have to be integrated. This is much more difficult and has rarely been achieved. Intercultural cooperation is typically impeded by tribal prejudice. In Tanzania, for example, there are about 120 different tribes, speaking 108 different languages. While they have lived together relatively peaceful since independence, members of one tribe typically have misconceptions about other tribes. Cooperation is usually hampered by mistrust and anxiety. If methods can be found that support confidencebuilding, ease of involvement and equal participation of different communities, this will not only help to preserve cultural identities, but will also assist in solving intertribal conflicts and other community-related problems that hinder development.

In many developing countries, there are often enormous inequalities in power and wealth, resulting partly from the continuation of feudalistic structures. In African countries, the 'power distance', i.e. the degree of centralization of authority and autocratic leadership (Hofstede, 1983), is still large and widespread. Chieftainship has been one of the few stable and enduring institutions of the African society (Roberts, 1968) which has remained autocratic because its members permitted it and its leaders saw no reason to give up their privileges. There is no doubt that this power distance 
aggravated development.

One of its particularly negative effects has been its influence on maintaining the inequalities between male and female participation in education. Improving education for girls may be the most important element of development policy in Africa. It is 'a powerful cause of reduced fertility' (WorldBank, 1992). Women who have been able to complete a secondary education have, on average, only three instead of seven children. In Tanzania, for example, which shares with Malawi the lowest gross secondary school enrollment rate of only $5 \%$, the percentage of female students in upper secondary school is only about $25 \%$. The University enrollment rate of women has steadily declined during the last ten years from approximately $26 \%$ of all students to $17 \%$ (Mlama, 1995). Development chances will inevitably be adversely affected by this.

During the last ten years, development agencies have found that only approaches to decisionmaking that provided procedures to maximize involvement and participation of stakeholders led to acceptable planning and implementation of development projects. For long-term sustainable development, techniques and tools to enhance a participative culture, to minimize power distances, to foster development-orientation, and to reinforce equality and democratic procedures are of paramount importance. Without broad-based cooperation and sharing of responsibilities tangible development success will not be achieved.

Analogous to findings about resistance to implementation of information systems, one can assume that failure of development projects can be prevented by avoiding clashes between cultural presumptions and an actually existing culture (Pliskin, et al., 1993). Such clashes can be minimized and perhaps excluded completely by methods and tools that support cooperation by reducing inequalities, facilitating participation in decision-making, blocking domination or discrimination of individuals or minorities, and help to create a development-conducive atmosphere of tolerance and appreciation of cultural diversity.

\subsection{Economic aspects}

\section{Harnessing the 'Information revolution'}

While the idea of the 'information revolution' has almost become trivial among scholars, it was only in February 1995, that the first ministerial meeting of the G7 group of industrialized countries (Britain, Canada, France, Germany, Italy, Japan, USA) on the global information society took place. In their communique, the ministers agreed that 'the smooth and effective transition towards the information society is one of the most important tasks that should be undertaken in the last decade of the 20th century'. Governments seem to have realized now that IT is an essential development factor.

In an attempt to assist developing countries to 'harness the information revolution', i.e. make better use of IT for poverty alleviation and sustainable economic development, the World Bank has recently revised some of its objectives in this area and plans to give more support for:

- widespread and equitable access to communication and information services through accelerated deployment of national information infrastructure and effective integration into international communication and information networks;

- systemic improvements in the functioning and competitiveness of key economic sectors through strategic information policies and systems;

- new ways to use IT to help solve the most pressing problems of human and economic development - education, health, poverty alleviation, rural development, and care for the environment (Talero/Gaudette, 1995). 
The emerging information society is characterized by substantial differences from the industrial society, such as more competition, more democracy, less centralization, etc. In the information society, 'trade and investments are global and organizations compete with knowledge, networking and agility on a global basis' (Talero/Gaudette, 1995). To cope with these changes requires structural adjustments, including regulatory and institutional reforms in all countries. Many developing countries are still hesitant or opposed to such reforms. They may not realize that they 'risk exclusion from the global economy and severe competitive disadvantages for their goods and services' (Talero/Gaudette, 1995), if they do not accept to reform their policies.

\section{Productivity and competitiveness}

Competitiveness and smooth organizational performance are critical success factors in an ever more closely linked global economy. They are also traditional weaknesses in many developing countries, particularly in African societies. It is obvious that this has contributed to the increasing marginalization of Africa within the last two decades.

Successful integration of developing countries into the world's communication networks requires adequate IT solutions which, in turn, represent an opportunity to accelerate development through:

- increased competitiveness for local industries in international markets,

- increased business opportunities through connections to trading networks,

- increased participation in research and development for local scientists,

- productivity increases in government services,

- major improvements in the delivery of services, such as education, health, agricultural extension, management of the environment, promotion of the private sector, etc.

Despite these prospects, only few governments have embarked on a systematic exploitation of IT for development. Particularly in Sub-Saharan Africa, IT strategies and national informatics policies are lacking.

Slow acceptance of IT has partly also been observed in industrialized countries and has been explained by discouraging results from analyzing previous IT investments. Several studies in the US came to the conclusion that the widespread use of IT had not resulted in tangibly increasing office productivity. One of the main reasons for that was the fact that most IT applications were simply automated versions of existing business processes. These processes had not been re-engineered before computerization. They reflected all weaknesses that existed before automation. IT cannot be blamed for this lack of strategic information systems planning. The observed lack of productivity increase, sometimes called the 'productivity paradoxon' of IT investment, has also been caused by:

- a generally insufficient use of IT for strategic decision-making,

- a typical underrepresentation of IT on the top level of an organization, with a chief information officer being an exception rather than the rule, and

- tall hierarchies in governmental and large corporate organizations with their extraordinary need for communication, coordination and control measures consuming many of the positive impacts of IT.

These observations demonstrate a rather limited validity of the productivity paradoxon of IT. Without adequate IT investment, developing countries will lessen their chances for productivity increases. More than any other technology, IT will prevent further loss of competitiveness and will help to reintegrate developing countries into the world economy. 


\section{Structural adjustments and reforms}

On the macroeconomic level, structural adjustments have become a centerpiece of development and a prerequisite for development assistance. Without agreement on reforms of the public sector and the civil service, new multilateral loans are rarely given to governments in developing countries and even bilateral aid has been made dependent on such adjustments.

On the microeconomic level, structural reforms are equally required. Traditionally, corporate structures have been hierarchical. In recent years, however, more and more organizations have realized that obvious changes in human behavior require adequate structural adjustments as well. Reduced willingness to defer to authority and more demand for involvement in decision-making has contributed to flattening management structures and greater autonomy for staff (Lewis, 1994).

Hierarchical systems have proved unsuitable for rapid adaptation to changes required in a more competitive business environment. They cannot respond quickly enough to change because of the long time communication takes up and down the chain of command (Pava, 1982). Competitive organizations are characterized by high flexibility and responsiveness. Small, self-managed groups have been best in achieving such goals. Business re-engineering has during the last few years had a strong impact on creating such groups of managers and experts, collaborating through appropriate IT systems.

It is likely that the increase of group work will change the way corporations are organized and may accelerate the gradual 'demise of hierarchies'. There will be a shift of emphasis from financial to human capital (McNurlin/Sprague, 1989). Problems are becoming more complex and cannot longer be solved by individual managers or specialists. Teams must be formed since no one person has enough knowledge, experience or information. Organizations are becoming more dependent on workgroups to design problem solutions, take decisions and carry out the basic activities to meet organizational goals (Lewis, 1994). Workgroups will probably be an important part of an emerging organizational culture in the 'International Office of the Future' (Glasson, 1994) and will influence economic and social development anywhere in the world.

\section{STRUCTURE AND PROCESSES OF DEVELOPMENT DECISION CENTERS}

\subsection{Goals and objectives}

While the enormous difficulties of sustainable and human development for which there is no onedimensional solution cannot be underrated, the view presented in this paper is that most problems are man-made and can be overcome through better decision-making and application of appropriate strategies. The strategy suggested here is the establishment of DDC within community information centers. DDC are expected to:

- accelerate development by decentralization and provision of facilities for demand-oriented, community-initiated and locally managed development programs

- enhance strategic development planning and decision-making capabilities by making optimal use of the emerging organizational culture of workgroups

- develop a culture of participative decision-making by enabling and improving approaches that minimize power distance and inhibit discrimination and domination of minorities

- improve the quality of decision-making, group productivity and motivation

- enable access to essential information services and offer new ways to solve development problems by educating decision-makers in IT based methods and tools 
- contribute to a future-oriented information infrastructure

- support the concept of 'good governance' through enforcing democratic procedures, transparency and accountability

- assist in harnessing the 'information revolution' for development and enhancing competitiveness

- function as a hub for integrating developing countries into international communication, trade and cooperation networks

- reduce rural-urban migration.

A prototype of a DDC, providing an example for an 'electronic meeting room' that could be established within a community information center in a rural or urban area of a developing country, has been built up in the Faculty of Commerce and Management of the University of Dar es Salaam (UDSM). It serves the university community, government agencies, public and private organizations, and provides assistance for management and other groups of decision-makers in the process of developing, discussing and evaluating goals, plans, projects, strategies, etc. as well as access to decision-oriented information. The overall goal of the UDSM DDC is to help improve group decision-making through the application of adequate IT. Main objectives are to facilitate strategic decision-making, train decision-makers, enable research in group support and information systems.

\subsection{Hardware and software configuration}

The UDSM DDC is equipped with nine microcomputers, i.e. eight notebook PCs for the participants and one server. The participants' workstations have 80386 CPUs, 1 MB RAM and a VGA display. The chauffeur station has a Pentium CPU with 8 MB RAM, a fast harddisk drive and an SVGA display.

Notebook PCs have been chosen for the participants as these could be integrated into tables that have been designed to accommodate two computers each. The tables are arranged in U-shape which is well-suited for face-to-face discussions. The computers are placed in drawers and are thus less obtrusive, as the displays can be folded down and the drawers can be pushed back into the tables when not needed during a session. The drawers also contain the network cables connecting the participants' computers to the 'chauffeur' workstation which serves as the network controller and file server. The chauffeur workstation is also used to compute the results of the group work and display them through a video projector onto a common screen.

The group support system (GSS) used is called 'MeetingWorks for Windows'. It is based on ideas developed in the first doctoral dissertation on group decision support systems by Lewis (Lewis, 1982) who further developed it until 1992, and was then rewritten for the 'Windows' environment by Enterprise Solutions of Seattle (Enterprise Solutions, 1994). The system is designed for small groups engaged in face-to-face meetings. It is presently used at about 30 universities and between 60 and 100 public and private organizations in the US, Canada, Australia, New Zealand and Germany. The installation at the University of Dar es Salaam is the first in a developing country.

The GSS requires a network-based file system for communication between the chauffeur and the participants' workstations. The file system must support standard 'DOS' and 'Windows' file access over the network. In the UDSM DDC, 'Lantastic' has been installed for that purpose and configured for shared access in such a way that each station can read and write files on the network server disk, where the chauffeur files are installed. 


\subsection{Functions and results of DDC-based development decision-making}

\section{Meeting preparation}

Preparing a development decision-conference or session requires thorough planning. This process is supported by the SCRYPTWRITING module which enables the facilitator of a meeting to define the meeting steps and allocate the appropriate tools to these steps, thus creating a suitable agenda. Figure 1 shows a script for a strategic development planning process, generated with this module. Preliminary session steps, such as clarifying the purpose and scope of the session and cxplaining the background and restrictions, as well as the creation of the final report of the meeting results are done without using the GSS. As the system is run under 'Windows', these steps can be supported by other 'Windows'-based software, such as a spreadsheet and wordprocessing system.

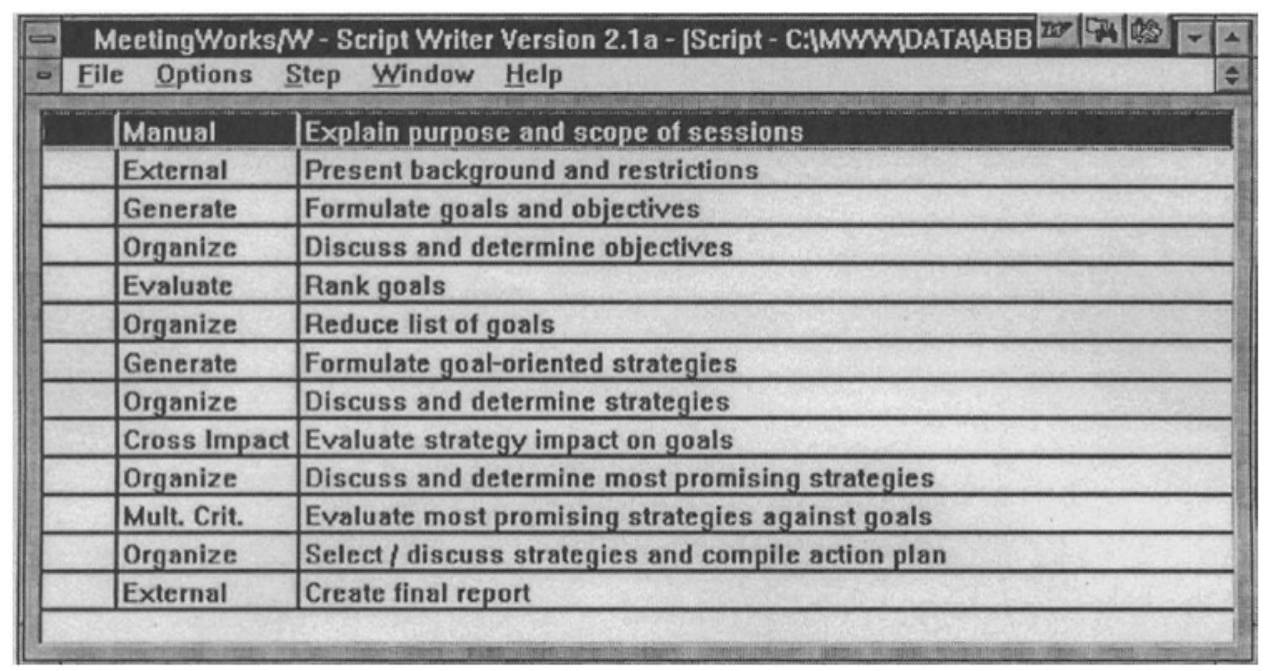

Figure 1 Script for a strategic development planning process

\section{Idea generation}

Stralegic development planning typically starts with a brainstorming process. This is supported by the GENERATE modulc which ensures anonymily and thus protects minority viewpoints. All ideas entered into the participants' workstations are sent to a common file and displayed on the main screen at the front of the room, where everybody can read them (sec Figure 2) without knowing who contributed the idea. Participants can use 'piggybacking' for maximum crealivity. To avoid 'tunnel vision', which may occur if participants wait for one member to give some direction or guidance by entering his idcas first, switching on the front screen can bc delayed until everyone has entered some ideas. With this approach, one has the advantage of initial independent idea generation, while retaining the possibility for 'piggybacking' later in the process. This also helps to overcome 'dispositional anxiousness' that may affect the generation of ideas when brainstorming in groups (Camacho/Paulus, 1995).

Brainstorming in the DDC is supported by providing access to internal and external databases 
through connections to the UDSM network and the Internet.

\section{Idea discussion}

On completion of a brainstorming session, a group typically proceeds to discussing and cditing of the ideas generated. The DISCUSS module reads the list of items from a file created by GENERATE. Il displays one item at a time and manages a timed discussion of the items, using the shared screen at the front of the room (see Figure 3). The group may set an appropriate amount of time to consider each item which is displayed along with the remaining discussion time. The timer can be interrupted at any time to either allow more discussion time or to proceed to the next item. The system, thus, helps to allocatc each item adequate time and to avoid the frequently observed situation that the first few items of a list are discussed at length, and the ones at the bottom reccivc inadequate or no attention at all.

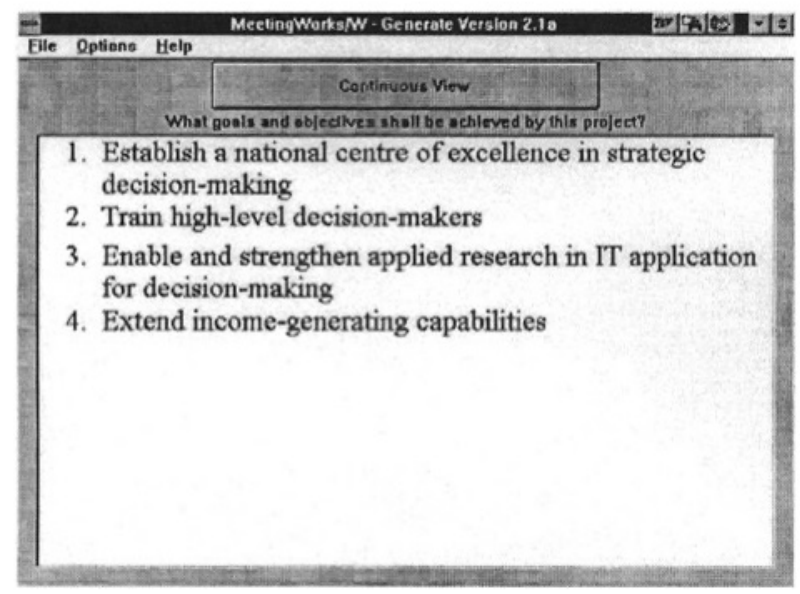

Figure 2 Common screen displaying brainstorming results.

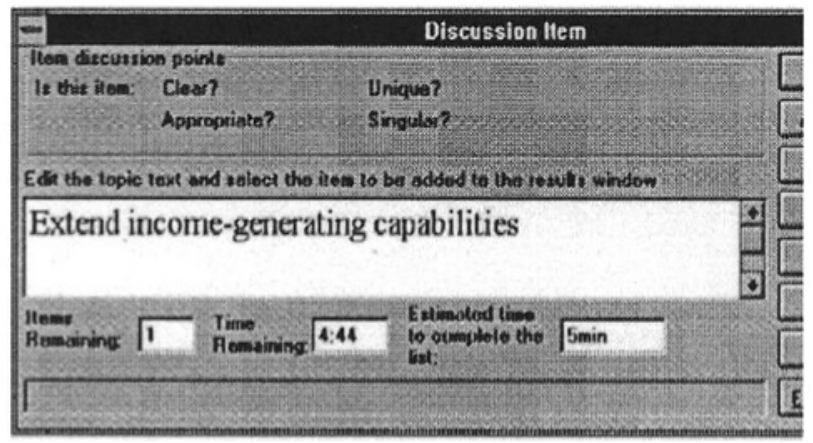

Figure 3 Discuss screen. 


\section{Idea organization}

If a group has developed a complex list of ideas, where it is important to structure the list, the ORGANIZE module assists in building outlines. Different levels of an outline can be defined and displayed on the front screen, and ideas can be placed in the appropriate location in the outline following discussion by the group (sce Figure 4).

The DISCUSS and ORGANIZE modules are usually run at the same time. The DISCUSS window displays the list of ideas, while the ORGANIZE window displays the outline. As each item comes up for discussion, it is eniered into the outline at a position detcrmined by the group.

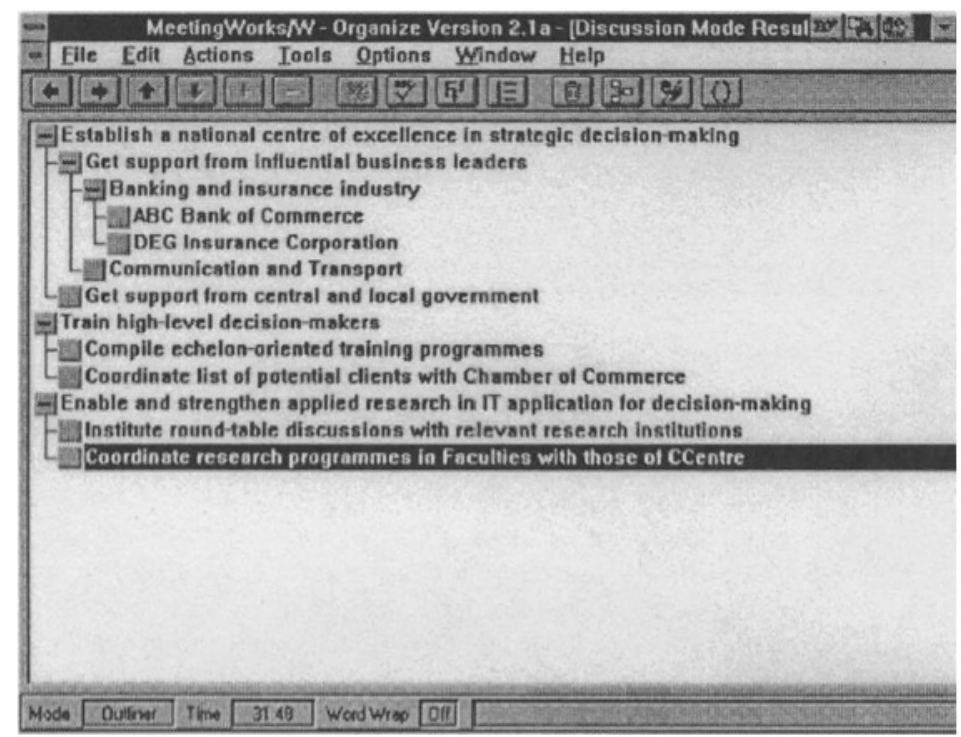

Figure 4 Organize screen

\section{Item evaluation}

lo prioritize or decide on the items of a list, an evaluation tool is required. The software provides several modules for this purpose, such as CROSS IMPACT ANALYSIS, VOTE, RANK, RATE, MULTIPLE CRITERIA ANALYSIS. All of these modules guarantee that evaluations made by the particjpants remain anonymous and cannot be used against any individual or group. 


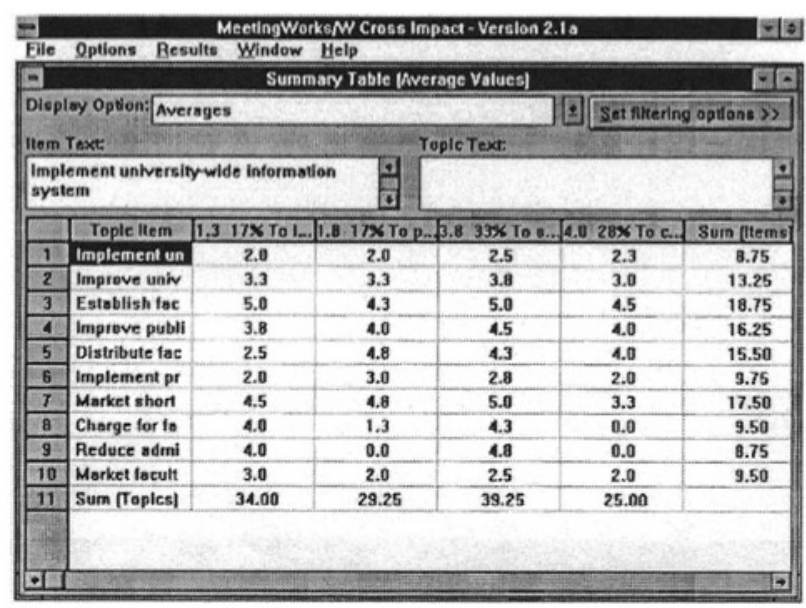

Figure 5 Results of a Cross Impact Analysis.

CROSS IMPACT ANALYSIS is used to compare any two lists of items. For example, a group may want to compare to what extent alternative strategies can help to achieve certain development goals, in order to prepare an appropriate shortlist for a linal evaluation with multiple criteria analysis. The module allows participants to make YES/NO decisions or to rate items on a scale from +5 to -5 . Figure 5 shows a table created by a Cross Impact Analysis of 10 strategies whose impact on 4 goals was evaluated. Based on the displayed results, it was decided that only those strategics with a total score of higher than 13 would be considered in the final evaluation process.

The RANK and RATE modules are similar in design and support a decision-maker in expressing preference for a certain itcm. The RANK module enables a participant to arrange the iterns in rank order, e.g. to allocate rank 1 to the goal or alternative he/she prefers or considers most important, etc. The RATE modulc allows participants to indicate their preferences on a 1 to 5,1 to 7 , or 1 to 10 scale. By using this program rather than the RANK module, decision-makers can express their opinion that there are two or more alternatives deserving the same judgment.

Each module provides a summary of the evaluation, indicating the average values and the variability as a percentage of the maximum standard deviation. The variability indicates agreement/disagreement among the participants. Figure 6 shows the results of a ranking decision. 


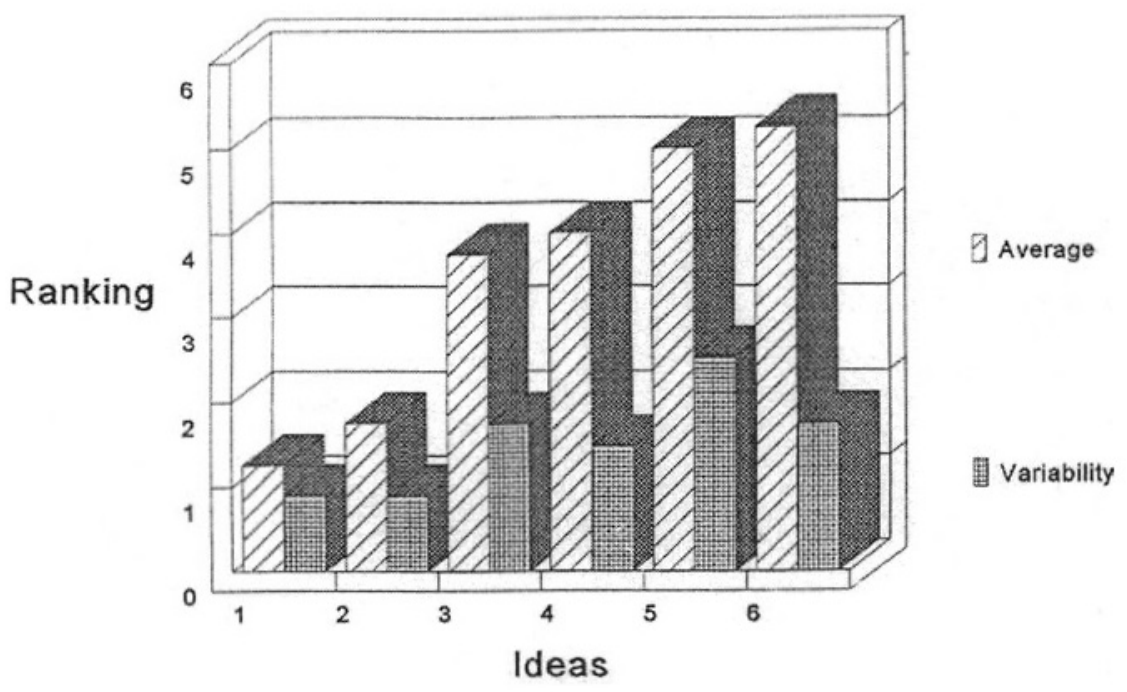

Figure 6 Results of a Ranking of Development Goals.

A useful feature for further analyzing agrcement/disagreement is the possibility to display the distribution of the decision-makers rankings (see Figure 7). The distribution graph shows a separate small graph for each item evaluated. The Y-axis represents the total number of participants who assigned the same value for an item. The $\mathrm{X}$-axis shows the actual values assigned. For example, the upper left graph indicates that the considered goal was ranked first by 3 participants and second by 1 participant.

The MULTIPLE CRITERIA ANALYSIS module is a multi-attribute tool, allowing a group to evaluate alternatives, for example development strategies, using a number of predetermined criteria. Each criterion is weighted by the group, i.e. it is assigned the average value of the individually assigned weights. Each participant rates how well each alternative meets each criterion. The product of the weight and rating determines the score for a given alternative on a given criterion. The scores are summed across all criteria for each alternative. The alternative with the highest score is considered the best. The individual evaluations are integrated into a results table and a final results graph, summarizing the mean of the participants' scores (see Figure 8).

The results window offers a number of options to further analyze the findings. By choosing the analysis method, the group can decide whether the 'group mean analysis' or the 'individual data analysis' method should be applied to compute the final results. By highlighting cells in the results table and clicking them on, detail bar graphs are displayed. Each of the displayed bars can be further analyzed with a mouse click. A detail graph may, for example, display the distribution of participant weighted scores for one alternative and one criterion. With another mouse click on each of the bars comments that may have been entered by the participants to explain their rationale for a particular rating can be displayed. Various filtering options can be used to show subsets of the results based on average values, variability or both. Weights and scores can also be easily re-evaluated. 


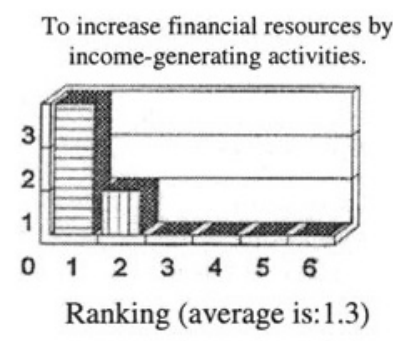

To strive for a relative

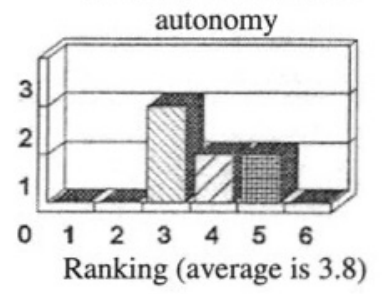

To improve organizational structure and processes in the faculty

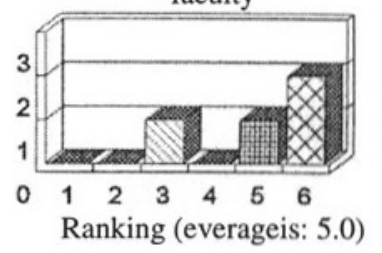

To promote linkage with all sectors of the economy

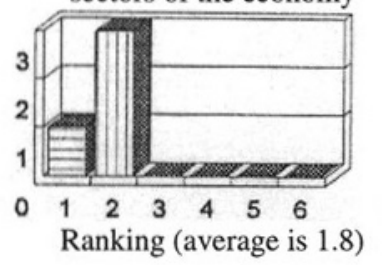

To conduct research and publish results

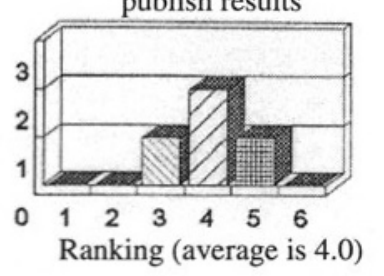

Preserve, enhance and transmit knowledge in subject area

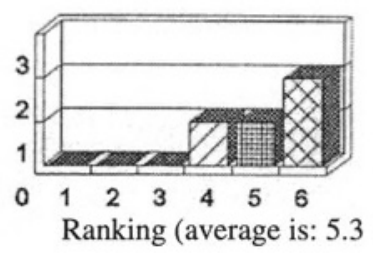

Figure 7 Distribution of ranking of 6 development goals by 4 participants.

At the end of a decision meeting, the results of the group's deliberations are typically summarized in a report. The system supports this process by providing a template, designed to make use of the MSWORD wordprocessor. A shell document containing macros to access the generated text and graphics files can be prepared. By integrating a report-writing phase into the script, the wordprocessor and the shell document are loaded. Running the macros then converts the shell into a professionally formatted report which can be ready for distribution within minutes of the completion of a session. Decision-makers will in this way not only receive a complete documentation of the group's work, but also a high-quality report including all tables and graphs produced during the course of the meeting. 


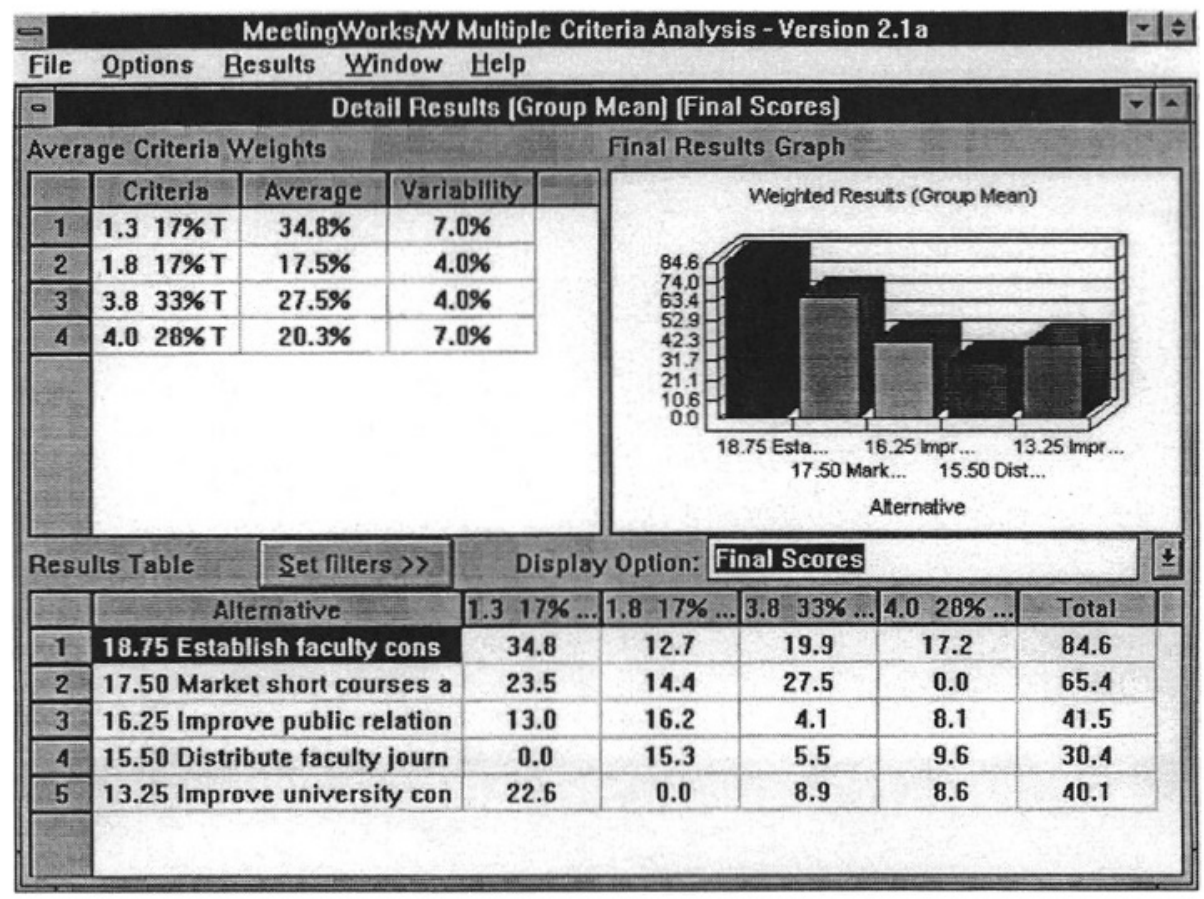

Figure 8 Results of a multiple criteria analysis of development strategies

\section{BENEFITS OF DEVELOPMENT DECISION CENTERS}

DDC can be seen as an example of 'culture-centered computing' (Hakken, 1991), particularly when installed within community information centers. They provide a strategy to enhance intercultural understanding and are likely to enhance creativity and originality of development planning. In a study involving mixed groups of introverts and extroverts and using traditional face-to-face meetings and a GSS to discuss scenarios, it was found that all participants contributed more original solutions using the GSS (Yellen et al, 1995). This is consistent with earlier findings in a different environment (Petrovic, 1993).

When established within institutions of higher learning, government agencies, public and private corporations, etc., to offer facilities for solving complex problems that require close collaboration of experts and policy-makers, DDC can contribute to making organizations more effective and efficient. Equipped with non-obtrusive hardware and with group support systems they achieve these improvements through the typical characteristics of computer-supported group work:

- Parallel and anonymous input of ideas, preferences, evaluations;

- Focussed discussion and easy organization of texts;

- Problem orientation instead of people orientation;

- Automatic preference analysis;

- Application of more sophisticated evaluation procedures;

- Automatic report generation. 
DDC help to enhance strategic development planning and decision-making capabilities by making optimal use of human resources and IT. They improve the quality of decision-making in groups, as well as group productivity and motivation. They contribute to a culture of participative policymaking by minimizing power distance and preventing inequalities, discrimination and domination of minorities.

When integrated into community information centers, they help to create a future-oriented information infrastructure, facilitate access to essential information services and offer new methods to solve development problems. They provide a means to educate decision-makers in IT-based techniques and tools and support the concept of 'good governance' through enforcing democratic procedures, transparency, and accountability. They ensure that residents, non-governmental organizations, and businesses in rural and urban areas receive economical and easy access to service systems and networks for training, technical assistance, marketing, banking, government licensing, etc. This appears particularly relevant for small and medium enterprises that are recognized as 'vital engines' (Talero/Gaudette, 1995) of economic development and job creation. In this manner, they help to eliminate the typical development inequalities between rural and urban areas, and contribute to more evenly spread development chances.

As there seems to be strong evidence of a positive correlation between IT infrastructure and the level of development (Kraemer, 1992), it is likely that the combination of community information centers and DDC will contribute to accelerate development and to increase a country's participation in the world economy. This, in turn, is likely to have positive effects on the domestic growth rate (Talero/Gaudette, 1995) as well.

DDC assist in harnessing the 'information revolution' for development. For that purpose, developing countries frequently have to make structural adjustments, requiring substantial capital investments, whose benefits may take years or even decades to materialize. DDC, on the other hand, are innovations that need only very moderate investment and achieve an immediate positive impact on development decision-making and IT infrastructure. They help to integrate developing countries into international communication, trade and cooperation networks, and are likely to reduce migration to urban centers as they improve rural living and development conditions.

DDC can gather and disseminate development-relevant knowledge and best practices. They contribute to rationalize development planning and facilitate integration of the developing countries into the 'information society'. By providing methods and tools to accelerate decisionmaking and to generate comprehensive documentation of decision processes, they promote the idea of 'direct democracy' and challenge the typical bureaucratic rigidity and lack of responsiveness of public administration. In this manner, they also support political stability in developing countries.

\section{REFERENCES}

Aiken, M., Kim, D., Hwang, C., Lu, L.C. (1995) A Korean Group Decision-Support System. INFORMATION \& MANAGEMENT, 28, 5, 303-310.

Blunt, P (1995), Cultural Relativism, Good Governance and Sustainable Human-Development. PUBLIC ADMINISTRATION AND DEVELOPMENT, 15, 1, 1-9.

Camacho, L.M. and Paulus, P.B. (1995) The Role of Social Anxiousness in Group Brainstorming. JOURNAL OF PERSONALITY AND SOCIAL PSYCHOLOGY, 68, 6, 1071-1080.

Enterprise Solutions Inc. (1994) MeetingWorks for Windows. The Technology of Consensus. Seattle. 
Glasson, B.C. (1994) International Office of the Future: A Teaching and Research Facility, in APPLICATIONS AND IMPACTS, Information Processing '94, Proceedings of the IFIP 13th World Computer Congress, Hamburg, Germany, 28 August - 2 September, Amsterdam, NorthHolland, 430-435.

Hakken, D. (1991) Culture-Centered Computing - Social-Policy and Development of New Information Technology in England and the United-States. HUMAN ORGANIZATION, 50, 4, 406-423.

Hofstede, G. (1983) The Cultural Relativity of Organizational Practices and Theories, Journal of International Business Studies, 14, 2, 75-89.

Kraemer, K., Gurbaxani, V., King, J. (1992) Economic Development, Government Policy, and the Diffusion of Computing in Asia-Pacific Countries, Public Administration Review, $52,2$.

Lewis, L.F. (1982) Facilitator: A Microcomputer Decision Support System for Small Groups. Unpublished doctoral dissertation, University of Louisville.

Lewis, L.F. (1994) A Brief Introduction to Group Support Systems. MeetingWorks Associates, Bellingham.

McNurlin, B.C. and Sprague, R.H. (1989) Information Systems Management in Practice, 2nd edition. Prentice Hall, Englewood Cliffs, New Jersey.

Pava, C. (1982) Microelectronics and the Design of Organization, Working Paper No. HBS 82-67, Harvard Business School, Division of Research, Soldier Field, Boston, Mass.

Petrovic, O. and Krickl, O. (1993) Traditionell-moderiertes vs. computergestütztes Brainstorming. Eine vergleichende Betrachtung. Wirtschaftsinformatik, 2, 120-128.

Pliskin, N., Romm, T., Lee, A.S., Weber, Y., (1993) Presumed Versus Actual Organizational Culture - Managerial Implications for Implementation of Information-Systems, COMPUTER JOURNAL, 36, 2, 143-152.

Roberts, A. (ed) (1968) Tanzania before 1900. East African Publishing House, Nairobi.

Talero, E. and Gaudette, P. (1995) Harnessing Information for Development. The World Bank, Washington.

WorldBank, (1992) World Development Report 1992. Development and the Environment. Oxford University Press, New York.

Yellen, R.E., Winniford, M., Sanford, C.C. (1995) Extroversion and Introversion in ElectronicallySupported Meetings, INFORMATION \& MANAGEMENT, 28, 1, 63-74.

\section{BIOGRAPHY}

Dietrich Splettstoesser is a Senior Lecturer in the Faculty of Commerce and Management of the University of Dar es Salaam, Tanzania. He holds an MBA degree from the University of Cologne, Germany, and a PhD degree from the University of Linz, Austria.

He has worked as consultant, lecturer, and manager in Europe, Australia and Africa, and has more than 25 years of experience in developing and implementing information systems. Since 1981 he has advised national and international organizations in Kenya, Uganda, and Tanzania, and has managed the development and implementation of several large-scale information systems. 